\title{
NEW ESTIMATES FOR SMOOTH WEYL SUMS
}

\author{
TREVOR D. WOOLEY
}

\section{Introduction}

Since the early part of this century, estimates for Weyl sums (or generalisations thereof) have been central to the treatment of many problems in the additive theory of numbers. For over forty years, the strongest such estimates have stemmed from a method due to Vinogradov [8], the argument having been somewhat simplified recently by the use of the large sieve (see [4, Lemma 5.4]). During this period, improvements in estimates for generalisations of Weyl sums have arisen from improved bounds on mean values of such sums, very recently with the arrival of Vaughan's new iterative method (see [5, Theorems 1.5 and 1.8]). In contrast, this paper will be devoted to improvements at the core of this circle of ideas, within Vinogradov's method itself. Our ideas, which here we shall investigate in the context of smooth Weyl sums, would seem to be applicable elsewhere, and this is a matter which we intend to pursue in the future. We now describe our conclusions in some detail.

Let $k$ be a natural number, and $P$ be a large real number. When $2 \leqslant R \leqslant P$, we define the set of $R$-smooth numbers, $\mathscr{A}(P, R)$, by

$$
\mathscr{A}(P, R)=\{n \in[1, P] \cap \mathbb{Z}: p \text { prime, } p \mid n \Rightarrow p \leqslant R\},
$$

and for each real number $\alpha$, we define the corresponding smooth Weyl sum, $f(\alpha ; P, R)$, by

$$
f(\alpha ; P, R)=\sum_{x \in \mathscr{A}(P, R)} e\left(\alpha x^{k}\right)
$$

where here, and throughout, we write $e(\alpha)=e^{2 \pi i \alpha}$. The precise form of our results is to be found in Section 4 , the following upper bound being a simple corollary.

THEOREM 1.1. Let $m$ denote the set of real numbers $\alpha$ such that whenever $a \in \mathbb{Z}$, $q \in \mathbb{N},(a, q)=1$ and $|\alpha-a / q| \leqslant q^{-1} P^{1-k}$, one has $q>P$. Then when $\eta=\eta(\varepsilon, k)$ is $a$ sufficiently small positive number, and $2 \leqslant R \leqslant P^{\eta}$, we have

$$
\sup _{\alpha \in \mathfrak{m}}|f(\alpha ; P, R)| \ll_{\varepsilon, k} P^{1-\rho(k)+\varepsilon},
$$

where, when $k$ is large, $\rho(k)^{-1}=k(\log k+O(\log \log k))$.

For comparison, Vaughan [5, Theorem 1.8] obtains a similar result with the exponent satisfying $\rho(k)^{-1}=(4+o(1)) k \log k$, this having been improved, by means of 
superior mean value estimates, by Wooley [9, Theorem 1.4] to the extent that ' 4 ' can be replaced by ' 2 ' in the latter conclusion. We note that when $R=P$, the exponential sum $f(\alpha ; P, R)$ represents a classical Weyl sum, for which the best estimate corresponding to Theorem 1.1 currently has exponent satisfying $\rho(k)^{-1}=$ $(2+o(1)) k^{2} \log k$ (see [10, Corollary 1.1]). Thus, since card $\left(\mathscr{A}\left(P, P^{\eta}\right)\right) \gg_{\eta} P$ when $\eta$ is positive, for points in $m$, estimates for smooth Weyl sums are considerably sharper than those for classical Weyl sums.

As far as applications of our new estimate in additive number theory are concerned, we shall restrict ourselves to a cursory consideration of two basic problems. First we improve localised estimates for the fractional parts of $\alpha n^{\kappa}$.

THEOREM 1.2. Let $k \in \mathbb{N}, \alpha \in \mathbb{R}$ and $\varepsilon>0$. Then there is a real number $N(\varepsilon, k)$ such that whenever $N \geqslant N(\varepsilon, k)$, we have

$$
\min _{1 \leqslant n \leqslant N}\left\|\alpha n^{k}\right\| \leqslant N^{\varepsilon-\tau(k)}
$$

where, when $k$ is large, $\tau(k)$ satisfies $\tau(k)^{-1}=k(\log k+O(\log \log k))$.

This may be compared with [11, Corollary to Theorem 1.2], where a similar result is established with $\tau(k)^{-1}=2 k(\log k+\log \log k+2+o(1))$. Our improvements in Waring's problem are, unfortunately, rather small. This is because improvements in 'minor arc' estimates for exponential sums tend to have a less significant impact, within the circle method, than reduced upper bounds for mean values. As usual, we define $G(k)$ to be the smallest number $s$ such that every sufficiently large natural number is the sum of, at most, $s k$ th powers of natural numbers. The precise form of our new estimate (see Corollary 1 to Theorem 4.2) leads to the following bounds for $G(k)$ when $10 \leqslant k \leqslant 20$.

THEOREM 1.3. We have $G(10) \leqslant 62, G(12) \leqslant 78, G(13) \leqslant 86, G(14) \leqslant 94$, $G(15) \leqslant 102, G(16) \leqslant 110, G(17) \leqslant 118, G(18) \leqslant 127, G(19) \leqslant 135, G(20) \leqslant 144$.

For comparison, the respective bounds $G(10) \leqslant 63, G(12) \leqslant 79, G(13) \leqslant 87$, $G(14) \leqslant 95, G(15) \leqslant 103, G(16) \leqslant 112, G(17) \leqslant 120, G(18) \leqslant 129, \quad G(19) \leqslant 138$, $G(20) \leqslant 146$ were obtained in [9, Theorem 1.1]. When $k=11$, our improvements are not sufficiently large to lead to a visible reduction in $G(k)$. For large $k$ our methods now lead to the following upper bound on $G(k)$.

\section{THEOREM 1.4. We have}

$$
G(k) \leqslant k\left(\log k+\log \log k+2+O\left(\frac{\log \log k}{\log k}\right)\right) .
$$

This conclusion may be compared with [11, Theorem 1.3], where a similar result is given with ' $2+\log 2$ ' replacing ' 2 '.

Our estimate for $f(\alpha ; P, R)$ is based on an application of the large sieve inequality, in essence, at least in the initial stages of the argument, following the treatment of 
Vaughan [5, Section 10]. We let $M$ be a real number with $P^{1 / 2} \leqslant M \leqslant P$ to be chosen later. By Dirichlet's Theorem, we may find $a \in \mathbb{Z}$ and $q \in \mathbb{N}$ with $(a, q)=1$, $|q \alpha-a| \leqslant \frac{1}{2}(M R)^{-k}$ and $q \leqslant 2(M R)^{k}$. Then, by a suitable combinatorial lemma combined with Hölder's inequality, when $s \in \mathbb{N}$ we are able to bound the smooth Weyl sum $f(\alpha ; P, R)$ in the form

$$
f(\alpha ; P, R)^{2 s} \ll M^{2 s+\varepsilon}+(M R)^{2 s-1} \sum_{\substack{M<v \leqslant M R \\(v, q)=1}}\left|\sum_{1 \leqslant y \leqslant s(P / M)^{k}} b_{y} e\left(\alpha v^{k} y\right)\right|^{2},
$$

where $b_{y}$ denotes the number of solutions of the diophantine equation

$$
u_{1}^{k}+\ldots+u_{s}^{k}=y
$$

with $u_{i} \in \mathscr{A}(P / M, R)$ for $1 \leqslant i \leqslant s$. We may classify the values of $v$ into $O\left(P^{\varepsilon}\right)$ sets $\mathscr{V}_{1}, \ldots, \mathscr{V}_{L}$ so that $v_{1}, v_{2} \in \mathscr{V}_{j}$ and $v_{1}^{k} \equiv v_{2}^{k}(\bmod q)$ together imply that $v_{1} \equiv v_{2}(\bmod q)$. Thus, provided that $M$ is chosen suitably, there is a $j$ satisfying

$$
\left.\left.f(\alpha ; P, R)^{2 s} \ll(M R)^{2 s-1+\varepsilon} \sum_{v \in \mathscr{V}_{j}}\right|_{1 \leqslant y \leqslant s(P / M)^{k}} b_{y} e\left(\alpha v^{k} y\right)\right|^{2} .
$$

When $\alpha$ lies in a set of type similar to the set $m$ defined in the statement of Theorem 1.1 , an analysis of the $\alpha v^{k}$ shows that they are spaced at least $(2 q)^{-1}$ apart modulo 1 . Then by the large sieve inequality,

$$
f(\alpha ; P, R)^{2 s} \ll(M R)^{2 s-1+\varepsilon}\left(q+s(P / M)^{k}\right) \sum_{1 \leqslant y \leqslant Y} b_{y}^{2}
$$

the last sum being bounded by using a suitable mean value estimate for smooth Weyl sums. The strength of the ensuing bound for $f(\alpha ; P, R)$ now depends on the relative magnitudes of $M$ and $q+(P / M)^{k}$, our estimate improving as the former parameter grows and the latter expression decreases. Vaughan takes $M=P^{1 / 2}$, which leads to the bound $q+(P / M)^{k} \ll P^{k / 2}$. We take $M=P^{\lambda}$ with $\lambda$ a parameter satisfying $\frac{1}{2}<\lambda<1$. As it stands, it is possible that $q$ is as large as $P^{k \lambda}$, and this would lead to weak bounds on $f(\alpha ; P, R)$. However, by modifying an argument of Heath-Brown [2, Section 5], it is possible to obtain a complementary bound on $f(\alpha ; P, R)$ which is useful only when $q$ is large. Thus we are able to discard those $q$ with $q>P^{k(1-\lambda)}$, and thereby achieve a suitable bound on $q+(P / M)^{k}$.

The above argument is effective for the set of $\alpha$ satisfying the property that whenever $a \in \mathbb{Z}, q \in \mathbb{N},(a, q)=1$ and $|\alpha-a / q| \leqslant q^{-1} M P^{-k}$, one has $q>M$. In Vaughan's analysis one is constrained to take $M \leqslant P^{1 / 2}$, which limits the strength of the ensuing bounds. The basic advantage of our new method is the relaxation of this condition, and indeed for large $k$ we may now take $M=P^{1+o(1)}$. We note that Thanigasalam [3] has obtained a variant of Vinogradov's method which can be applied to smooth exponential sums (see [5, Section 9]). The latter method permits one to take $M=P^{k /(2 k-1)}=P^{\frac{1}{2}+O(1 / k)}$, so that it is asymptotically of no greater strength than that due to Vaughan.

In Section 2 we record some basic estimates for mean values of smooth Weyl sums, and also provide a suitable combinatorial lemma for our later arguments. Section 3 is devoted to the task of establishing an asymmetric estimate for $f(\alpha ; P, R)$ 
of value for large moduli $q$, which in combination with a suitable estimate for small moduli in Section 4, leads to the desired estimate for $f(\alpha ; P, R)$. In Sections 5 and 6 we then draw corollaries concerning Waring's problem and the fractional parts of polynomials.

\section{Preliminary observations}

We start by recalling some of the salient features of the new iterative method in Waring's problem. Throughout, $s, t$ and $u$ will denote positive integers, and $\varepsilon$ and $\eta$ will denote sufficiently small positive numbers. We take $P$ to be a large positive real number depending at most on $k, s, t, u, \varepsilon$ and $\eta$. We use $\ll$ and $\gg$ to denote Vinogradov's well-known notation, implicit constants depending at most on the latter numbers. Also, we write $[x]$ for the greatest integer not exceeding $x$, and write $\|x\|$ for $\min _{y \in Z}|x-y|$. In order to simplify our analysis, we adopt the following convention concerning the numbers $\varepsilon$ and $R$. Whenever $\varepsilon$ or $R$ appear in a statement, either implicitly or explicitly, we assert that for each $\varepsilon>0$, there exists a positive number $\eta_{0}=\eta_{0}(\varepsilon, s, t, u, k)$ such that the statement holds whenever $R=P^{\eta}$, with $0<\eta \leqslant \eta_{0}$. Note that the 'value' of $\varepsilon$, and $\eta_{0}$, may change from statement to statement, and hence also the dependency of implicit constants on $\varepsilon$ and $\eta$.

We define $S_{8}(P, R)$ to be the number of solutions of the diophantine equation

$$
x_{1}^{k}+\ldots+x_{s}^{k}=y_{1}^{k}+\ldots+y_{s}^{k},
$$

with $x_{i}, y_{i} \in \mathscr{A}(P, R)$ for $1 \leqslant i \leqslant s$. Thus

$$
S_{s}(P, R)=\int_{0}^{1}|f(\alpha ; P, R)|^{2 s} d \alpha .
$$

We shall say that an exponent $\Delta_{s}=\Delta_{s, k}$ is permissible whenever the exponent has the property that $S_{s}(P, R) \ll P^{\lambda_{s, k}+\varepsilon}$, with $\lambda_{s, k}=2 s-k+\Delta_{s, k}$. It follows easily that any permissible exponent $\Delta_{\varepsilon, k}$ is non-negative, and moreover, without loss of generality, that $\Delta_{s, k} \leqslant k$. The following lemma provides us with permissible exponents when $k$ is large.

LeMMA 2.1. Let $k \geqslant 4$ and $t \in \mathbb{N}$. For each $s \in \mathbb{N}$ with $2 \leqslant s \leqslant t$, define the real number $\Delta_{s}=\Delta_{s, k}$ to be the unique positive solution of the equation $\Delta_{s} e^{\Delta_{8} / k}=k e^{1-28 / k}$. Then $\Delta_{s, k}$ is permissible, and consequently the exponent $\Delta_{s, k}^{*}=k e^{1-2 s / k}$ is permissible.

Proof. This is the corollary to [11, Theorem 2.1], which simplifies [9, Lemma 3.2].

We shall require a result on the density of integers with a given square-free kernel. Given an integer $v$ with canonical prime factorisation $\prod_{i=1}^{t} p_{i}^{r_{i}}$, we denote by $s_{0}(v)$ the square-free kernel of $v$, that is, $\prod_{i=1}^{i} p_{i}$. Furthermore, we define the set $\mathscr{C}_{q}(Q)$ by

$$
\mathscr{C}_{Q}(Q)=\left\{x \in \mathbb{Z} \cap[1, Q]: s_{0}(x) \mid s_{0}(q)\right\}
$$

LEMMA 2.2. Suppose that $L$ is a positive real number and $r$ is a positive integer with $\log r \ll \log L$. Then for each $\varepsilon>0$, $\operatorname{card}\left(\mathscr{C}_{r}(L)\right) \ll L^{e}$.

Proof. For each divisor $d$ of $r$, it follows from [9, Lemma 2.1] that

$$
\text { card }\left\{1 \leqslant y \leqslant L: s_{0}(y)=s_{0}(d)\right\} \ll L^{\varepsilon} .
$$


The lemma now follows by using standard estimates for the divisor function.

We conclude this section by providing a means of decomposing the smooth Weyl $\operatorname{sum} f(\alpha ; P, R)$ into a form in which we can apply the arguments of Sections 3 and 4 . For this purpose we apply essentially the same argument as that used by Vaughan [5, p. 67].

LEMMA 2.3. Let $\alpha \in \mathbb{R}$ and $r \in \mathbb{N}$, and suppose that $Q, M$ and $R$ satisfy $2 \leqslant R \leqslant M<Q$. Then

$$
\sum_{\substack{x \in \mathscr{A}(Q, R) \\(x, r)-1}} e\left(\alpha x^{k}\right) \ll R \log Q \max _{\substack{\pi \leqslant R \\ \pi \text { prime }}} \sup _{\theta \in[0,1)} V_{r}(\alpha ; Q, M, R ; \pi, \theta)+M,
$$

where

and

$$
V_{r}(\alpha ; Q, M, R ; \pi, \theta)=\sum_{\substack{v \in \mathscr{Q}(M, \pi, R) \\(v, r)-1}}\left|\sum_{\substack{u \in \mathscr{S}(Q / M, \pi) \\(u, r)-1}} e\left(\alpha(u v)^{k}+\theta u\right)\right|,
$$

$$
\mathscr{B}(M, \pi, R)=\{v \in \mathbb{N}: M<v \leqslant M \pi, \pi \mid v, \text { and p prime, } p \mid v \Rightarrow \pi \leqslant p \leqslant R\}
$$

Proof. On following the argument leading to equation (10.9) of Vaughan [5], it is apparent that the conclusion remains valid with first summation in equation (10.10) adjusted so that the condition $M<v \leqslant M R$ is replaced by $v \in \mathscr{B}(M, p, R)$. The lemma therefore follows immediately from [5, (10.4), (10.6), (10.9) and (10.10)].

\section{Upper bounds for large moduli}

When $\alpha$ is close to a rational $a / q$ with $q$ large, we use an asymmetric form of an argument similar to one used by Heath-Brown [2, Section 5] in the estimation of the fractional part of $\alpha n^{k}$.

LEMMA 3.1. Suppose that $\lambda$ satisfies $\frac{1}{2}<\lambda<1$, and write $M=P^{\lambda}$. Let $\alpha \in \mathbb{R}$, and suppose that there exist $a \in \mathbb{Z}$ and $q \in \mathbb{N}$ satisfying $(a, q)=1$ and $|\alpha-a / q| \leqslant q^{-2}$. Then when $t, w \in \mathbb{N}$, and $\Delta_{t}$ and $\Delta_{w}$ are permissible,

$$
f(\alpha ; P, R) \ll q^{\ell} P^{1+\varepsilon}\left(M^{\Delta_{w}}(P / M)^{\Delta_{t}}\left(q^{-1}+M^{-k}+(P / M)^{-k}+q P^{-k}\right)\right)^{1 / 2 t w}+M .
$$

Proof. By applying Lemma 2.3 with $r=1$, we deduce that there exists a prime $\pi$ with $\pi \leqslant R$, and $\theta \in[0,1)$ such that

$$
f(\alpha ; P, R) \ll P^{\varepsilon} R \sum_{v \in \mathscr{A}(M R, R)}|h(\alpha ; v, \theta)|+M,
$$

where $h(\alpha ; v, \theta)=\sum_{u \in \mathscr{A}(P / M, \pi)} e\left(\alpha(u v)^{k}+\theta u\right)$. Define the complex numbers of unit modulus, $\varepsilon(v, \theta)$, by

$$
|h(\alpha ; v, \theta)|^{t}=\varepsilon(v, \theta) h(\alpha ; v, \theta)^{t} .
$$

Also, let $r_{c}$ denote the number of solutions of the diophantine equation

$$
u_{1}^{k}+\ldots+u_{t}^{k}=c
$$


with $u_{i} \in \mathscr{A}(P / M, \pi)$, in which each solution $\mathbf{u}$ is counted with weight $e\left(\theta\left(u_{1}+\ldots+u_{t}\right)\right)$. Thus $h(\alpha ; v, \theta)^{t}=\sum_{1 \leqslant c \leqslant t(P / M)^{k}} r_{c} e\left(\alpha c v^{k}\right)$, and hence an application of Hölder's inequality, together with (3.2), yields

$$
\begin{aligned}
\left(\sum_{v \in \mathscr{A}(M R, R)}|h(\alpha ; v, \theta)|\right)^{t} & \leqslant(M R)^{t-1} \sum_{v \in \mathscr{A}(M R, R)}|h(\alpha ; v, \theta)|^{t} \\
& =(M R)^{t-1} \sum_{1 \leqslant c \leqslant t(P / M)^{k}} r_{c} \sum_{v \in \mathscr{A}(M R, R)} \varepsilon(v, \theta) e\left(\alpha c v^{k}\right) .
\end{aligned}
$$

Next we note that $\left|r_{c}\right| \leqslant n_{c}$, where $n_{c}$ denotes the number of solutions of the equation (3.3) with $u_{i} \in \mathscr{A}(P / M, \pi)$. A further application of Hölder's inequality therefore shows that

$$
\left(\sum_{v \in \mathscr{A}(M R, R)}|h(\alpha ; v, \theta)|\right)^{2 t w} \leqslant(M R)^{2 w(t-1)}\left(\sum_{c} n_{c}\right)^{2 w-2}\left(\sum_{c} n_{c}^{2}\right) J_{w}(\alpha),
$$

where

and

$$
J_{w}(\alpha)=\sum_{1 \leqslant c \leqslant t(P / M)^{k}}|g(\alpha ; c, \theta)|^{2 w},
$$

$$
g(\alpha ; c, \theta)=\sum_{v \in \mathscr{A}(M R, R)} \varepsilon(v, \theta) e\left(\alpha c v^{k}\right)
$$

But by considering the underlying diophantine equations, we have $\sum_{c} n_{c} \leqslant(P / M)^{t}$ and $\sum_{c} n_{c}^{2} \leqslant S_{t}(P / M, R)$, and hence

$$
\left(\sum_{v \in \mathscr{A}(M R, R)}|h(\alpha ; v, \theta)|\right)^{2 t w} \leqslant(P R)^{2 t w}(P / M)^{-2 t}(M R)^{-2 w} J_{w}(\alpha) S_{t}(P / M, R) .
$$

Let $\tilde{n}_{d}$ denote the number of solutions of the equation $\sum_{i=1}^{w} v_{i}^{k}-\sum_{i=w+1}^{2 w} v_{i}^{k}=d$, with $v_{i} \in \mathscr{A}(M R, R)$ for $1 \leqslant i \leqslant 2 w$, each solution $\mathbf{v}$ counted with weight $\prod_{i-1}^{w} \varepsilon\left(v_{i}, \theta\right) \overline{\varepsilon\left(v_{w+i}, \theta\right)}$. Then by considering the underlying diophantine equation,

$$
\tilde{n}_{d}=\int_{0}^{1}|g(\beta ; c, \theta)|^{2 w} e(-\beta c d) d \beta
$$

Therefore, on making a trivial estimate, $\left|\tilde{n}_{d}\right| \leqslant \tilde{n}_{0} \leqslant S_{w}(M R, R)$, since $|\varepsilon(v, \theta)|=1$. On recalling equation (3.4), we have

$$
\begin{aligned}
J_{w}(\alpha) & =\sum_{1 \leqslant c \leqslant t(P / M)^{k}} \sum_{|\alpha| \leqslant w(M R)^{k}} \tilde{n}_{d} e(\alpha c d) \leqslant S_{w}(M R, R) \sum_{|\alpha| \leqslant w(M R)^{k}}\left|\sum_{1 \leqslant c \leqslant t(P / M)^{k}} e(\alpha c d)\right| \\
& \ll S_{w}(M R, R) \Upsilon(q),
\end{aligned}
$$

where

$$
\Upsilon(q)=\sum_{|d| \leqslant w(M R)^{k}} \min \left\{(P / M)^{k},\|\alpha d\|^{-1}\right\}
$$

But by using [4, Lemma 2.2] we obtain

$$
\Upsilon(q) \ll(P R)^{k+\varepsilon} q^{\ell}\left(q^{-1}+(P / M)^{-k}+M^{-k}+q P^{-k}\right) .
$$


Therefore, by (3.5), (3.6), and recalling the discussion of $S_{s}(P, R)$ in Section 2, we deduce that

$$
\begin{aligned}
\sum_{v \in \mathscr{A}(M R, R)}|h(\alpha ; v, \theta)| & \ll\left((P R)^{2 t w+\varepsilon}(P / M)^{-k+\Delta_{t}}(M R)^{-k+\Delta_{w}} \Upsilon(q)\right)^{1 / 2 t w} \\
& \ll q^{\varepsilon} P^{1+\varepsilon}\left((P / M)^{\Delta_{t}} M^{\Delta_{w}}\left(q^{-1}+(P / M)^{-k}+M^{-k}+q P^{-k}\right)\right)^{1 / 2 t w}
\end{aligned}
$$

The lemma now follows immediately from (3.1).

\section{Upper bounds for small moduli}

When $\alpha$ is close to a rational $a / q$ with $q$ small, we are able to adapt a variant of Vinogradov's method given by Vaughan [5, Section 10] to provide an upper bound for $f(\alpha ; P, R)$. Our proof will differ in detail from that of Vaughan, since we are able to provide some technical simplifications which lead to a more precise result.

LEMMA 4.1. Suppose that $\frac{1}{2}<\lambda<1$, and write $M=P^{\lambda}$. Let $\alpha \in \mathbb{R}$, and suppose that $a \in \mathbb{Z}$ and $q \in \mathbb{N}$ satisfy $(a, q)=1,|q \alpha-a| \leqslant \frac{1}{2}(M R)^{-k}, q \leqslant 2(M R)^{k}$ and either $|q \alpha-a|>M P^{-k}$ or $q>M R$. Then if $s$ is a natural number with $2 s \geqslant k+1$, and $\Delta_{s}$ is permissible,

$$
f(\alpha ; P, R) \ll M^{1+\varepsilon}+P^{1+\varepsilon}\left(M^{-1}(P / M)^{\Delta_{s}}\left(1+q(P / M)^{-k}\right)\right)^{1 / 2 s} .
$$

Proof. Observe that

$$
f(\alpha ; P, R)=\sum_{x \in \mathscr{A}(P, R)} e\left(\alpha x^{k}\right)=\sum_{d \in \mathscr{Q}_{q}(P) \cap \mathscr{A}(P, R)} \sum_{\substack{x \in \mathcal{A}(P / d, R) \\(x, q)-1}} e\left(\alpha(x d)^{k}\right) .
$$

Thus, on applying Lemma 2.2,

$$
\begin{aligned}
f(\alpha ; P, R) & \ll \sum_{d \in \mathscr{Q}_{q}(P / M)}\left|\sum_{\substack{x \in \mathcal{Q}(P / d, R) \\
(x, q)-1}} e\left(\alpha(x d)^{k}\right)\right|+\sum_{\substack{d \in \mathscr{Q}_{q}(P) \\
d>P / M}} P / d \\
& \ll P^{e} \max _{d \in \mathbb{Q}_{q}(P / M)}\left|\sum_{\substack{x \in \mathcal{O}^{\alpha}(P / d, R) \\
(x, q)-1}} e\left(\alpha(x d)^{k}\right)\right|+M^{1+\varepsilon}
\end{aligned}
$$

Next, by Lemma 2.3 , it follows that for some $d \in \mathscr{C}_{q}(P / M), \theta \in[0,1)$, and prime $\pi \leqslant R$, we have

$$
f(\alpha ; P, R) \ll M^{1+\varepsilon}+P^{e} R g(\alpha ; d, \pi, \theta),
$$

where

$$
g(\alpha ; d, \pi, \theta)=\sum_{\substack{v \in Q(M / d, \pi, R) \\(v, q)=1}}\left|\sum_{\substack{u \in \mathscr{S}(P / M, \pi) \\(u, q)-1}} e\left(\alpha(u v d)^{k}+\theta u\right)\right|
$$

Notice here that $M / d \geqslant M^{2} / P=P^{2 \lambda-1}$, so that $M / d$ is at least as large as some positive power of $P$.

Let $J(q, d, h)$ denote the number of solutions of the congruence $(x d)^{k} \equiv h(\bmod q)$ with $1 \leqslant x \leqslant q$. Then when $(h, q) \mid d^{k}$, a simple estimate gives $J(q, d, h) \ll q^{\varepsilon} d^{k}$. Hence there is an $L \ll q^{e} d^{k}$ such that the $v$ with $M / d<v \leqslant M R / d$ and $(v, q)=1$ can be divided into $L$ classes $\mathscr{V}_{1}, \ldots, \mathscr{V}_{L}$ such that for any two distinct elements $v_{1}, v_{2}$ in a given 
class $\mathscr{V}_{j}$, we have $\left(v_{1} d\right)^{k} \equiv\left(v_{2} d\right)^{k}(\bmod q)$ if and only if $v_{1} \equiv v_{2}(\bmod q)$. On writing $c_{y}$ for the number of solutions of the diophantine equation $u_{1}^{k}+\ldots+u_{s}^{k}=y$ with $u_{i} \in \mathscr{A}(P / M, \pi)$, we may apply Hölder's inequality to obtain

$$
g(\alpha ; d, \pi, \theta)^{2 s} \ll P^{\varepsilon} d^{k}(M R / d)^{2 s-1} \max _{1 \leqslant j \leqslant L} \sum_{v \in \mathscr{V}_{j}}\left|\sum_{1 \leqslant y \leqslant s(P / M)^{k}} b_{y} e\left(\alpha(v d)^{k} y\right)\right|^{2},
$$

where $\left|b_{y}\right| \leqslant c_{y}$.

Now recall that $|q \alpha-a| \leqslant \frac{1}{2}(M R)^{-k}$. Thus, if $v_{1}, v_{2} \in \mathscr{V}_{j}$ and $v_{1} \not \equiv v_{2}(\bmod q)$, then we have $\left(v_{1} d\right)^{k} \not \equiv\left(v_{2} d\right)^{k}(\bmod q)$, and hence

$$
\left\|\alpha\left(\left(v_{1} d\right)^{k}-\left(v_{2} d\right)^{k}\right)\right\| \geqslant\left\|\frac{a}{q}\left(\left(v_{1} d\right)^{k}-\left(v_{2} d\right)^{k}\right)\right\|-\frac{1}{2} q^{-1} \geqslant \frac{1}{2} q^{-1} .
$$

We divide into cases.

(i) Suppose that $q>M R / d$. Then the elements of $\mathscr{V}_{j}$ are distinct modulo $q$, and hence it follows from (4.3) that for $v \in \mathscr{V}_{j}$, the $\alpha(v d)^{k}$ are spaced at least $\frac{1}{2} q^{-1}$ apart modulo 1.

(ii) Suppose that $q \leqslant M R / d$. Then since $q \leqslant M R$, by hypothesis we have $|q \alpha-a|>M P^{-k}$. Given any two distinct elements $v_{1}, v_{2}$ of $\mathscr{V}_{j}$ with $v_{1} \not \equiv v_{2}(\bmod q)$, we may conclude, as in Case (i), that $\alpha\left(v_{1} d\right)^{k}$ and $\alpha\left(v_{2} d\right)^{k}$ are spaced at least $\frac{1}{2} q^{-1}$ apart modulo 1 . Thus we are left to consider the situation in which $v_{1} \equiv v_{2}(\bmod q)$, but $v_{1} \neq v_{2}$. Then

$$
\left\|\alpha\left(\left(v_{1} d\right)^{k}-\left(v_{2} d\right)^{k}\right)\right\|=\left\|(\alpha-a / q)\left(\left(v_{1} d\right)^{k}-\left(v_{2} d\right)^{k}\right)\right\|=|\alpha-a / q| \cdot\left|\left(v_{1} d\right)^{k}-\left(v_{2} d\right)^{k}\right|
$$

Since $v_{1}-v_{2}$ is a non-zero multiple of $q$, and $v_{1} d>M$ and $v_{2} d>M$, we have

$$
\left\|\alpha\left(\left(v_{1} d\right)^{k}-\left(v_{2} d\right)^{k}\right)\right\| \geqslant|\alpha-a / q| M^{k-1} q>(P / M)^{-k} .
$$

Therefore, in this case, for $v \in \mathscr{V}_{j}$ the $\alpha(v d)^{k}$ are spaced at least $\frac{1}{2} \min \left\{q^{-1},(P / M)^{-k}\right\}$ apart modulo 1 .

Then in either case, by the large sieve inequality (see, for example, [4, Lemma 5.3]) we have

$$
\begin{aligned}
\left.\left.\sum_{v \in \mathscr{V}_{1}}\right|_{1 \leqslant y \leqslant s(P / M)^{k}} b_{y} e\left(\alpha(v d)^{k} y\right)\right|^{2} & \ll\left(q+(P / M)^{k}\right) \sum_{1 \leqslant y \leqslant s(P / M)^{k}}\left|b_{y}\right|^{2} \\
& \ll\left(q+(P / M)^{k}\right) S_{\delta}(P / M, R) .
\end{aligned}
$$

Then since $2 s \geqslant k+1$, it follows from (4.1) and (4.2) that

$$
f(\alpha ; P, R) \ll M^{1+\varepsilon}+\left(P^{\epsilon}(M R)^{2 s-1}(P / M)^{2 s-k+\Delta_{s}}\left(q+(P / M)^{k}\right)\right)^{1 / 2 s} .
$$

The lemma now follows immediately.

THEOREM 4.2. Suppose that $\frac{1}{2}<\lambda<1$. Let $\alpha \in \mathbb{R}$, and suppose that whenever $a \in \mathbb{Z}$ and $q \in \mathbb{N}$ satisfy $(a, q)=1$ and $|\alpha-a / q| \leqslant q^{-1} P^{\lambda-k}$, then one has $q>P^{\lambda} R$. Then if $s, t, w \in \mathbb{N}$ satisfy $2 s \geqslant k+1$, and $\Delta_{s}, \Delta_{t}$ and $\Delta_{w}$ are permissible exponents,

where

$$
f(\alpha ; P, R) \ll P^{e}\left(P^{\lambda}+P^{1-\mu(k)}+P^{1-v(k)}\right),
$$

$$
\mu(k)=\left(k(1-\lambda)-\lambda \Delta_{w}-(1-\lambda) \Delta_{t}\right) / 2 t w \quad \text { and } \quad v(k)=\left(\lambda-(1-\lambda) \Delta_{\delta}\right) / 2 s .
$$


Proof. Write $M=P^{\lambda}$. By Dirichlet's Theorem, there exist $a \in \mathbb{Z}$ and $q \in \mathbb{N}$ with $(a, q)=1, q \leqslant 2(M R)^{k}$ and $|q \alpha-a| \leqslant \frac{1}{2}(M R)^{-k}$. If $q>(P / M)^{k}$, then we may apply Lemma 3.1 to obtain the bound

$$
\begin{aligned}
f(\alpha ; P, R) & \ll P^{1+\varepsilon}\left(P^{\lambda \Delta_{w}+(1-\lambda) \Delta_{t}}\left(P^{-k(1-\lambda)}+P^{-k \lambda}\right)\right)^{1 / 2 t w}+P^{\lambda} \\
& \ll P^{\lambda+\varepsilon}+P^{1-\mu(k)+\varepsilon} .
\end{aligned}
$$

Thus we may assume that $q \leqslant(P / M)^{k}$. In this second case we apply Lemma 4.1 to establish the estimate

$$
f(\alpha ; P, R) \ll P^{\lambda+\varepsilon}+P^{1+\varepsilon}\left(P^{-\lambda+(1-\lambda) \Delta_{s}}\right)^{1 / 2 \varepsilon} \ll P^{\lambda+\varepsilon}+P^{1-v(k)+\varepsilon} .
$$

This completes the proof of the theorem.

By simply choosing $\lambda$ optimally in Theorem 4.2 , we obtain the following conclusion.

COROLlaRy 1 . Let $s, t, w$ be natural numbers satisfying $2 s \geqslant k+1$, and suppose that $\Delta_{n}$ for $n=s, t, w$ are permissible exponents. Define

$$
\sigma(k)=\frac{k-\Delta_{t}-\Delta_{s} \Delta_{w}}{2\left(s\left(k+\Delta_{w}-\Delta_{t}\right)+t w\left(1+\Delta_{s}\right)\right)} \quad \text { and } \quad \lambda(k)=\frac{s\left(k-\Delta_{t}\right)+t w \Delta_{s}}{s\left(k+\Delta_{w}-\Delta_{t}\right)+t w\left(1+\Delta_{s}\right)}
$$

Suppose that $\frac{1}{2}<\lambda(k)<1-\sigma(k)$. Then when $\alpha$ satisfies the hypotheses of Theorem 4.2,

$$
f(\alpha ; P, R) \ll P^{1-\sigma(k)+\varepsilon} .
$$

We now explore the consequences of our new estimate when $k$ is large.

COROllary 2. Let $\mathfrak{m}_{\lambda}$ denote the set of $\alpha \in \mathbb{R}$ such that whenever $a \in \mathbb{Z}, q \in \mathbb{N}$, $(a, q)=1$ and $|\alpha-a / q| \leqslant q^{-1} P^{\lambda-k}$, then $q>P^{\lambda} R$. Then there is a natural number $k_{0}(\varepsilon)$ with the following property. When $k \geqslant k_{0}(\varepsilon)$, there are real numbers $\lambda=\lambda(k)$ and $\sigma(k)$ with

$$
\frac{\log \log k}{\log k} \ll 1-\lambda \ll \frac{\log \log k}{\log k} \text { and } \sigma(k)^{-1}=k(\log k+O(\log \log k)),
$$

and such that

$$
\sup _{\alpha \in \mathrm{m}_{\lambda}}|f(\alpha ; P, R)| \ll P^{1-\sigma(k)+\varepsilon}
$$

Proof. We put

$$
s=\left[\frac{1}{2} k \log k\right], \quad t=\left[\frac{k \log \log k}{\log k}\right], \quad w=[k(\log \log k-\log \log \log k)] .
$$

Then on solving the equation $\xi e^{\xi}=e^{1-2 t / k}$, we find from Lemma 2.1 that the exponent $\Delta_{t}$ is permissible, where $\Delta_{t} / k=1-t / k+O\left(t^{2} / k^{2}\right)$. Hence

$$
k-\Delta_{t}=k \frac{\log \log k}{\log k}\left(1+O\left(\frac{\log \log k}{\log k}\right)\right) \text {. }
$$


Similarly, the exponents $\Delta_{w}$ and $\Delta_{s}$ are permissible, where by the concluding remark of Lemma 2.1,

$$
\Delta_{w}=k e^{1-2 w / k} \ll k\left(\frac{\log \log k}{\log k}\right)^{2} \quad \text { and } \quad \Delta_{s} \leqslant k e^{1-2 s / k} \ll 1 .
$$

We now note that, in the notation of Corollary 1 to Theorem 4.2,

$$
\sigma(k)^{-1}=2 s+\frac{2\left(s \Delta_{w}+t w\right)\left(1+\Delta_{s}\right)}{k-\Delta_{t}-\Delta_{s} \Delta_{w}} .
$$

Thus

$$
\sigma(k)^{-1}-k \log k \ll k \log \log k .
$$

Furthermore, again in the notation of Corollary 1 to Theorem 4.2,

$$
\lambda=1-\frac{s \Delta_{w}+t w}{s\left(k+\Delta_{w}-\Delta_{t}\right)+t w\left(1+\Delta_{s}\right)} .
$$

Thus

$$
\frac{\log \log k}{\log k} \ll 1-\lambda \ll \frac{\log \log k}{\log k},
$$

and so, in particular, when $k$ is sufficiently large we have $\frac{1}{2}<\lambda<1-\sigma(k)$, and so the hypotheses of Corollary 1 to Theorem 4.2 are satisfied. Therefore, in view of (4.4), the corollary follows immediately from Corollary 1 to Theorem 4.2. 4.2.

We note that Theorem 1.1 is merely a simplification of Corollary 2 to Theorem

\section{The estimation of $G(k)$}

The estimation of $G(k)$ is now relatively routine. It should be noted that our 'minor' arcs will be slightly different from those used in previous analyses (see, in particular, [6, Section 9]). Thus we shall take this opportunity to record the new results stemming from our analysis in the form of the following theorem.

THEOREM 5.1. Let $\lambda(k)$ and $\sigma(k)$ be the real numbers defined in Corollary 1 to Theorem 4.2. Under the same hypotheses as in that corollary, for each integer $v$ with $v \geqslant k-1$, and each permissible exponent $\Delta_{v}$, we have

$$
G(k) \leqslant \max \left\{2 v+3+\left[\frac{\Delta_{v+1}}{\sigma(k)}\right], 4 k\right\} .
$$

Proof. Let $n$ be a large positive number, and write $P=\left[n^{1 / k}\right]$. Let $v, w \in \mathbb{N}$ satisfy $v \geqslant k-1$, and write $s=2 v+w$. We consider the number of representations, $R(n)$, of $n$ in the form $n=x_{1}^{k}+x_{2}^{k}+y_{1}^{k}+\ldots+y_{s}^{k}$, with $x_{1}, x_{2} \in \mathbb{Z} \cap[1, P]$ and $y_{i} \in \mathscr{A}(P, R)$ for $1 \leqslant i \leqslant s$. On writing $g(\alpha)=\sum_{1 \leqslant x \leqslant P} e\left(\alpha x^{k}\right)$, we therefore have

$$
R(n)=\int_{0}^{1} g(\alpha)^{2} f(\alpha ; P, R)^{s} e(-\alpha n) d \alpha .
$$


Let $m$ denote the set of real numbers $\alpha \in[0,1)$ with the property that, whenever $a \in \mathbb{Z}$, $q \in \mathbb{N},(a, q)=1$ and $|\alpha-a / q| \leqslant q^{-1} P^{1-k}$, one has $q>P$. Then by Corollary 1 to Theorem 4.2, together with the remark at the end of [9, Section 3], it follows that

$$
\begin{aligned}
\int_{\mathrm{m}} g(\alpha)^{2} f(\alpha ; P, R)^{2 v+w} e(-\alpha n) d \alpha & \ll\left(\sup _{\alpha \in \mathrm{m}}|f(\alpha ; P, R)|\right)^{w} \int_{0}^{1}\left|g(\alpha)^{2} f(\alpha ; P, R)^{2 v}\right| d \alpha \\
& \ll\left(P^{1-\sigma(k)+\varepsilon}\right)^{w} P^{2 v+2-k+\Delta_{v+1}+\varepsilon} .
\end{aligned}
$$

Thus, provided that $w \sigma(k)>\Delta_{v+1}$, then

$$
\int_{m} g(\alpha)^{2} f(\alpha ; P, R)^{2 v+w} e(-\alpha n) d \alpha \ll P^{s+2-k-\delta}
$$

for some positive number $\delta$. The major arcs $\mathfrak{M}=[0,1) \backslash m$ may be dealt with by means of the same pruning argument as was used in [5, Section 5], owing to the presence of the factor $g(\alpha)^{2}$ (consisting of exponential sums over complete intervals). Thereby, one may obtain

$$
\int_{\mathfrak{M}} g(\alpha)^{2} f(\alpha ; P, R)^{2 v+w} e(-\alpha n) d \alpha \gg P^{s+2-k},
$$

and the desired conclusion follows immediately.

In order to prove Theorem 1.4, we have merely to apply Theorem 5.1 with $v=\left[\frac{1}{2} k(\log k+\log \log k+1)\right]$. From the concluding remark of Lemma 2.1 , the exponent $\Delta_{v+1}$ is permissible, where $\Delta_{v+1} \leqslant k e^{1-2(v+1) / k} \leqslant 1 / \log k$. Meanwhile, from Theorem 1.1 we have $\sigma(k)^{-1}=k \log k+O(k \log \log k)$. Thus, in Theorem 5.1 we deduce that

$$
G(k) \leqslant k(\log k+\log \log k+1)+k+O\left(\frac{k \log \log k}{\log k}\right)
$$

For Theorem 1.3 we must work a little harder. We calculate $\sigma(k)$ by applying Corollary 1 to Theorem 4.2 , using the values of $\Delta_{s}, \Delta_{t}$ and $\Delta_{w}$ listed in the table in the Appendix. The latter values of $\Delta_{n}$ may be shown, after some calculations, to be permissible by using the methods of [9] (we should point out that superior estimates should arise, albeit after much greater effort, from the methods of [7]). Having checked that the corresponding value of $\lambda(k)$, which is also listed in the table, satisfies the necessary hypotheses, we then apply Theorem 5.1 using the value of $v$ indicated in the Appendix. The estimates for $G(k)$ detailed in Theorem 1.3 then follow immediately.

\section{Localised estimates for fractional parts of polynomials}

We can dispose of the proof of Theorem 1.2 swiftly with a standard appeal to [1, Lemma 5]. We take $\lambda=\lambda(k)$ and $\sigma(k)$ to be as defined in the statement of Corollary 2 to Theorem 4.2. We let $\phi$ be any real number with $\varepsilon<\phi<\sigma(k)$. Let $P$ be a large positive number, and put $H=P^{o(k)-\phi}$. Define $T(\alpha)$ by

$$
T(\alpha)=\sum_{1 \leqslant h \leqslant H}|f(h \alpha ; P, R)| .
$$


Then provided that we can establish the bound $T(\alpha)=o(P)$, by [1, Lemma 5], as in [11, Section 5], it follows that $\min _{1 \leqslant n \leqslant P}\left\|\alpha n^{k}\right\|<P^{\phi-k}$.

Suppose first that there is a triple $h, a, q$ with $1 \leqslant h \leqslant H, a \in \mathbb{Z}, q \in \mathbb{N},(a, q)=1$, $|q h \alpha-a| \leqslant P^{\lambda-k}$ and $q \leqslant P^{\lambda} R$. Then

$$
\left\|\alpha(q h)^{k}\right\| \leqslant\left|(q h)^{k} \alpha-a(q h)^{k-1}\right|<\left(H P^{\lambda} R\right)^{k-1} P^{\lambda-k} \ll P^{-\sigma(k)} .
$$

Then in this case $\min _{1 \leqslant n \leqslant P}\left\|\alpha n^{k}\right\| \leqslant\left\|\alpha(q h)^{k}\right\|<P^{\phi-\sigma(k)}$.

In the alternative case, for each triple $h, a, q$ with $1 \leqslant h \leqslant H, a \in \mathbb{Z}, q \in \mathbb{N},(a, q)=1$ and $|q h \alpha-a| \leqslant P^{\lambda-k}$, we have $q>P^{\lambda} R$. Then by Corollary 2 to Theorem 4.2 ,

$$
\max _{1 \leqslant h \leqslant H}|f(h \alpha ; P, R)| \ll P^{1-\sigma(k)+\varepsilon} .
$$

Consequently, $T(\alpha) \ll H P^{1-\sigma(k)+\varepsilon}=o(P)$, and the desired conclusion follows once again.

\section{Appendix. Numerical values for parameters}

In this Appendix we display in tabular form the numerical values of the parameters arising in the method discussed in Section 5. The displayed figures were calculated to 12 significant figures on a computer, and then the values of the permissible exponents $\Delta_{n}$ and $\sigma(k)^{-1}$ were rounded up in the last displayed figure.

\begin{tabular}{ccccccccccc}
\hline$k$ & $s$ & $w$ & $t$ & $\Delta_{s}$ & $\Delta_{w}$ & $\Delta_{t}$ & $\sigma(k)^{-1}$ & $\lambda(k)$ & $v$ & $\Delta_{v+1}$ \\
\hline 10 & 22 & 13 & 7 & 0.22849 & 1.46021 & 4.06022 & 97.962 & 0.55161 & 25 & 0.09524 \\
11 & 25 & 15 & 8 & 0.22308 & 1.44899 & 4.32206 & 110.137 & 0.55358 & 29 & 0.08311 \\
12 & 28 & 16 & 9 & 0.22111 & 1.70161 & 4.59367 & 122.577 & 0.55521 & 32 & 0.09005 \\
13 & 31 & 18 & 9 & 0.22065 & 1.69429 & 5.45150 & 134.996 & 0.55702 & 35 & 0.09680 \\
14 & 35 & 20 & 10 & 0.19066 & 1.69881 & 5.71309 & 147.591 & 0.55847 & 39 & 0.08897 \\
15 & 38 & 21 & 11 & 0.19444 & 1.93796 & 5.97304 & 160.133 & 0.56014 & 43 & 0.08311 \\
16 & 41 & 22 & 12 & 0.19883 & 2.18309 & 6.23839 & 172.870 & 0.56153 & 46 & 0.08993 \\
17 & 44 & 24 & 12 & 0.20344 & 2.18066 & 7.09136 & 185.636 & 0.56296 & 50 & 0.08538 \\
18 & 47 & 26 & 13 & 0.20843 & 2.18618 & 7.35059 & 198.499 & 0.56436 & 54 & 0.08188 \\
19 & 51 & 27 & 14 & 0.19133 & 2.42272 & 7.60987 & 211.371 & 0.56567 & 57 & 0.08837 \\
20 & 54 & 29 & 14 & 0.19729 & 2.42874 & 8.46768 & 224.370 & 0.56682 & 61 & 0.08546 \\
\hline
\end{tabular}

Acknowledgement. The author thanks the referee for several useful comments which have improved the exposition of this paper.

\section{References}

1. G. Harman, 'Trigonometric sums over primes I', Mathematika 28 (1981) 249-254.

2. D. R. Heath-Brown, 'On the fractional part of $\alpha n^{k}$ ', Mathematika 35 (1988) 28-37.

3. K. Thanigasalam, 'Some new estimates for $G(k)$ in Waring's problem', Acta Arith. 42 (1982) 73-78.

4. R. C. Vaughan, The Hardy-Littlewood method (University Press, Cambridge, 1981).

5. R. C. Vaughan, 'A new iterative method in Waring's problem', Acta Math. 162 (1989) 1-71.

6. R. C. Vaughan and T. D. Wooley, 'On Waring's problem: some refinements', Proc. London Math. Soc. (3) 63 (1991) 35-68.

7. R. C. Vaughan and T. D. Wooley, 'Further improvements in Waring's problem', Acta. Math. to appear.

8. I. M. VInOgRadov, 'The method of trigonometrical sums in the theory of numbers', Trav. Inst. Steklov 23 (1947).

9. T. D. Wooley, 'Large improvements in Waring's problem', Ann. of Math. 135 (1992) 131-164.

10. T. D. WoOley, 'On Vinogradov's mean value theorem', Mathematika 39 (1992) 379-399. 
11. T. D. Wooley, 'The application of a new mean value theorem to the fractional parts of polynomials', Acta Arith., LXV. 2 (1993) 163-179.

Department of Mathematics

University of Michigan

Ann Arbor

Michigan 48109-1003

USA

E-mail: Trevor.Wooley@math.Isa.umich.edu 